\title{
Nota farmacológica: Tratamiento de la hipertensión arterial en la edad avanzada: menos es mejor
}

\author{
Treatment of elevated blood pressure in the very elderly. Less is better
}

Traducido con autorización de Therapeutics Initiative (The University of British Columbia). Disponible en URL: http://www.ti.ubc.ca/PDF/71.pdf

El riesgo de eventos adversos cardiovasculares graves aumenta con la edad avanzada y con la elevación de la presión arterial (PA). Sin embargo, la incidencia de caídas y otros efectos adversos graves asociados con la hipotensión postural también aumentan con la edad y con la terapia con medicamentos antihipertensivos. Por lo tanto, es importante saber si el tratamiento farmacológico no sólo mejora los resultados cardiovasculares, sino también medidas de beneficio sanitario neto que combinan beneficio y daño, como la mortalidad total o la incidencia de cualquier efecto adverso grave (véase Nota Terapéutica 42).

Esta "Nota Terapéutica" se centra en la mejor evidencia disponible sobre el tratamiento farmacológico de la hipertensión arterial en los individuos de 80 ó más años de edad.

\section{¿Cuál es la evidencia que respalda el tratamiento farma- cológico de la presión arterial elevada en pacientes de 60} a 80 años de edad?

Una revisión de la Colaboración Cochrane ${ }^{1}$ publicada en 1998 cuantificó los beneficios de la terapia farmacológica sobre la morbilidad y la mortalidad de los pacientes hipertensos de más de 60 años de edad con una PA sistólica igual o mayor a $160 \mathrm{mmHg}$.

Fueron seleccionados 15 ensayos controlados aleatorizados (ECA) de al menos un año de duración, que habían incluido a 21.908 pacientes, en su mayoría de 60 a 79 años de edad. La mayoría de los ensayos se realizaron en países industrializados occidentales, tuvieron tres a seis años de duración y evaluaron como primera línea de tratamiento a un diurético o un beta-bloqueante, con la posibilidad de reforzarlo agregando alguna otra clase de drogas.

Los resultados de este meta-análisis demostraron que cinco años de terapia farmacológica se asocian significativamente a una reducción de la mortalidad total (RR 0,88; IC95\% 0,82 a 0,$98 ; R R A=1,7 \%$; NNT=59) y a una reducción de un "resultado combinado" compuesto por la incidencia de accidente cerebrovascular (ACV) y de cardiopatía coronaria (RR 0,73; IC95\% 0,68 a 0,$77 ; R R A=5 \%, N N T=20$ ) sin brindar información sobre la incidencia del total de efectos adversos graves.

Por lo tanto concluimos que existe evidencia sólida y bien establecida para respaldar el tratamiento de los pacientes de 60 a 79 años con PA sistólica mayor o igual a 160 mmHg.

¿Cuál es la evidencia que respalda el tratamiento con drogas de los pacientes mayores de 79 años con elevación de la presión arterial?

Hasta el verano de 2008 la evidencia para el tratamiento de pacientes de 80 años o mayores se basaba principalmente en un metanálisis ${ }^{2}$ de subgrupo de varios ECA de la revisión Cochrane citada previamente.

Este metanálisis había sido publicado en 1999 y había incluido datos de 1670 participantes de 80 años o mayores, obtenidos a partir de siete ensayos. Este metanálisis encontró que el tratamiento de la hipertensión arterial se asocia a una reducción de la incidencia de ACV (RR 0,67; IC95\% 0,48 a 0,93) pero no de la mortalidad total (RR 1,09; IC95\% 0,95 a 1,25). Los autores concluyeron que, debido a que el tratamiento tiene un riesgo potencial de aumento de la mortalidad, es necesario contar con más ECA para avalar su implementación.
¿Qué hemos aprendido de los dos estudios HYVET?

En las investigaciones sobre la hipertensión en la edad avanzada (HYVET) los investigadores diseñaron dos ensayos para determinar si el tratamiento anti-hipertensivo era beneficioso en este grupo de mayor edad.

La investigación HYVET se inició con un ECA piloto multicéntrico abierto publicado en $2003^{3}$ y que había sido llevado a cabo en diez países europeos.

Habían participado 1.283 pacientes con una elevación sostenida de la PA sistólica de 160 a 219 y de la PA diastólica de 90 a $109 \mathrm{mmHg}$. Los pacientes fueron asignados al azar a uno de los tres tratamientos de primera línea: diuréticos tiazídicos, inhibidores de la enzima convertidora de angiotensina (IECA) o ningún tratamiento.

El objetivo fue que los pacientes mantuvieran una presión arterial menor a $150 / 80 \mathrm{mmHg}$, pudiendo duplicarse las dosis de las drogas de primera línea y añadirse diltiazem de liberación lenta. El seguimiento medio fue de 13 meses.

La mortalidad total de los pacientes del "HYVET piloto" no difirió en los dos brazos de tratamiento activo y cuando se la comparó con la ausencia de tratamiento, no mostró diferencias significativas (RR 1,23; IC95\%: 0,75 a 2,01).

El total de eventos adversos graves no fue informado y el total de ACV fue menor en las dos ramas de tratamiento activo que en la de los individuos no tratados (RR 0,50; IC95\%: 0,26 a 0,95).

Si bien estos resultados del "HYVET piloto" no respondieron la pregunta sobre el efecto del tratamiento con drogas sobre la mortalidad total, ayudaron en el diseño de los ECA oficiales publicados en agosto de $2008^{4}$.

El HYVET ${ }^{4}$ enroló 3.845 pacientes mayores de 80 años con una elevación sostenida de la PA sistólica por encima de 160 $\mathrm{mmHg}$, de 13 países de Europa Occidental y Oriental, China, Australasia y Norte de África. Los pacientes fueron aleatorizados a tratamiento activo o a un placebo idéntico.

El tratamiento activo consistió en análogos tiazídicos como 1,5 $\mathrm{mg}$ de indapamida de liberación sostenida (equivalente a aproximadamente $12,5 \mathrm{mg}$ de hidroclorotiazida) seguido por el IECA perindopril a una dosis desde $2 \mathrm{mg}$ para reforzar el tratamiento y hasta $4 \mathrm{mg}$ en caso de ser necesario. El objetivo terapéutico fue mantener la PA por debajo de $150 / 80 \mathrm{mmHg}$ y la mediana de seguimiento fue de 1,8 años.

El 26\% de los pacientes del grupo "tratamiento" pudo manejarse solamente con tiazidas, el $24 \%$ requirió diuréticos tiazídicos más 2 mg de perindopril y el $50 \%$ requirió tiazídicos más $4 \mathrm{mg}$ de perindopril. Se logró una PA menor a 150/80 mmHg en el $48 \%$ de las personas de la rama activa, y en el $20 \%$ de los asignados a placebo.

Los autores informaron los resultados HYVET a través del HR y en vez de la cantidad de personas con al menos un evento, reportaron el total de eventos. Por lo tanto, es imposible calcular el riesgo relativo o la reducción del riesgo absoluto, salvo para la mortalidad, para la que los pacientes pueden experimentar sólo una evento.

Este ensayo demostró que la terapia activa puede reducir un $18 \%$ la mortalidad total (RR 0,82; IC95\%: 0,69 a 0,99 ) y un $2,2 \%$, el riesgo absoluto (RRA) de muerte, necesitándose tratar 48 pacientes durante dos años (NNT) para evitar una muerte. Los autores también informaron el número de acontecimientos adversos graves como 358 en el grupo de tratamiento activo y 448 en el grupo placebo. El resultado primario (el total de ACV) no fue significativamente reducido (HR 0,70; IC95\%: 0,49 a 
$1,01)$ pero se redujo la incidencia de insuficiencia cardíaca (IC) mortal y de los episodios de IC no mortales (HR 0,36; IC95\%: $0,22$ a 0,58$)$.

La adición de estos dos estudios HYVET al metanálisis de subgrupos de la Colaboración Cochrane amplía la revisión a nueve ECA que comprenden 6.798 participantes con más de 80 años de edad al comienzo de los ensayos.

Los resultados de este nuevo metanálisis muestran que no hay efecto sobre la mortalidad total (RR 0,97; IC95\%: 0,87 a 1,08) pero sí una reducción significativa en el total de ACV (RR 0,67; IC95\%: 0,54 a 0,84). Esto se traduce en una reducción absoluta de $4 \%$ (RRA) del riesgo de ACV y la necesidad de tratar 25 personas de edad avanzada durante cinco años (NNT) años para evitar un evento. Cabe aclarar que en este grupo etario la incidencia de ACV a cinco años es de 12\% (incidencia en el grupo control en el ensayo para la hipertensión arterial sistólica en el adulto mayor ${ }^{2}$ para personas de 80 años o más).

\section{Interpretación clínica}

En general, la evidencia para reducir la presión arterial en las personas mayores de 80 años con presión sistólica mayor a $160 \mathrm{mmHg}$ surge de datos de pacientes relativamente sanos enrolados en ensayos clínicos. Por lo que consideramos que no es pertinente trasladarla a los ancianos enfermos o frágiles de la población general.

La evidencia combinada demuestra una reducción en la incidencia de ACV, pero no de la mortalidad total. Esto es una justificación razonable para ofrecer tratamiento con drogas a los ancianos hipertensos relativamente saludables, ya que estos pacientes quieren evitar los ACV de manera uniforme.
Si bien los resultados de los metanálisis, en general no pueden responder cual es la mejor opción de manejo con drogas, el ensayo más grande (HYVET ${ }^{4}$ y el único que ha demostrado un beneficio sanitario neto por haber encontrado una reducción concomitante de la mortalidad y del número de eventos adversos graves, proporciona un enfoque relativamente simple: comience con un diurético tiazídico en dosis bajas seguido de una dosis baja de un IECA.

Si fuera necesario para lograr una presión arterial menor a $150 / 80 \mathrm{mmHg}$ aumente una vez al doble la dosis del IECA. Mediante el uso de este enfoque se puede esperar que aproximadamente la mitad de los pacientes logre una presión arterial menor a $150 / 80 \mathrm{mmHg}$.

Este enfoque conservador del manejo de la presión arterial se ve corroborado por el reciente estudio observacional en individuos suecos mayores de 85 años, que sugiere que una presión arterial sistólica en un rango de 140 a 160 mmHg es óptima para los muy ancianos 5 .

\section{Conclusiones}

Para los pacientes mayores de 80 años:

- Diferentes tratamientos antihipertensivos de prevención primaria en pacientes relativamente saludables con presión sistólica mayor a $160 \mathrm{mmHg}$ redujeron los ACV pero no tuvieron efecto sobre la mortalidad total.

- En un gran ensayo clínico, el uso de bajas dosis de tiazidas como tratamiento de primera línea seguido por una dosis baja o media de IECA redujo la mortalidad, como así también los eventos adversos graves en un gran ECA.

- Con este régimen se puede esperar que alrededor del $50 \%$ de los pacientes alcance una presión arterial menor a 150/80 $\mathrm{mmHg}$.

La versión preliminar de esta nota terapéutica fue enviada para su revisión por 60 expertos y médicos de atención primaria para corregir cualquier inexactitud y asegurar que la información fuera concisa y relevante.

\section{Referencias}

1. Mulrow CD, Lau J, Cornell J, Brand M. Pharmacotherapy for hypertension in the elderly. Cochrane Database of Systematic Reviews 1998, Issue 2. Art No. : CD000028. DOI: 10.1002/14651858.CD000028.

2. Gueyffier F, Bulpitt C, Boissel, et al for the INDANA group. Antihypertensive drugs in very old people: a subgroup meta-analysis of randomized controlled trials. Lancet 1999; 353(9155):793-796.

3. Bulpitt CJ, Beckett NS, Cooke J, et al on behalf of the Hypertension in the Very Elderly Trial (HYVET)Working Group. Results of the pilot study for the Hypertension in the Very Elderly Trial. J of Hypertens 2003; 21: 2409-2417.

4. Beckett NS, Peters R, Fletcher AE, et al for the HYVET Study Group.Treatment of hypertension in patients 80 years of age or older. NEJM 2008;358(18): 1887-98.

5. Molander L, Lövheim H, Norman T, Nördstrom P, Gustafson Y. Lower systolic blood pressure is associated with greater mortality in people aged 85 and older. J Am Geriatr Soc 2008;56(10):1853-1859.

La nota terapéutica presenta un resumen de la evidencia obtenida de ensayos clínicos farmacológicos evaluados críticamente, que puede aplicarse a pacientes similares a los incluídos en esos ensayos pero no ser generalizable a cada paciente en particular. Nuestro objetivo es evaluar la efectividad de nuestras actividades educativas utilizando la red PharmaCare/PharmaNet, sin identificar médicos individuales, farmacias o pacientes. La Iniciativa Terpáutica es financiada por el Ministerio de Salud de British Columbia (BC) a través de un subsidio a la Universidad de BC. La Iniciativa Terapéutica provee consejos basados en la evidencia sobre terapéutica farmacológica, y no es responsable de la formulación o adjudicación de las políticas provinciales de medicamentos. 